\title{
Online Virtual Fit Is Not Yet Fit For Purpose: An Analysis Of Fashion e-Commerce Interfaces
}

\author{
Monika JANUSZKIEWICZ, Christopher J. PARKER, Steven G. HAYES, Simeon GILL \\ The University Of Manchester, Manchester, UK
}

DOI: $10.15221 / 17.210$ http://dx.doi.org/10.15221/17.210

\begin{abstract}
To unify the methodology of Virtual Fit platforms and allowing cross platform integration of 3D Body Scanning, the current Virtual Fit platforms need to be assessed in terms of their size recommendation approach and user interaction. Digital data, interactivity, and internet technology are changing the ways we interact in online shopping, with the Virtual Fit platforms having great potential to increase retail engagement and market share. This will support online purchasing activities while minimising the perceived risk in garment returns due to the poor sizing fit information.
\end{abstract}

Current research has focused on the analysis of computer modelling techniques, avatars, cloth, fabric draping simulations, and customer behaviour / aesthetic impact in the online domain. From a technical perspective, these investigations offer an interesting insight, although do not address issues of implementation or customer attitude. Therefore, to judge the current and potential impact of such technologies, it is important to understand 1) how they are being enacted online, 2) the Interaction Design elements of the user journey, 3) the application (or lack thereof) of mathematical models, and 4) how such interfaces are embedded within websites. Once these four key questions have been answered a greater understanding of how 3D Body Scanning and Technologies integrated into eCommerce and Virtual Fit platforms in the consumer market may be reached.

Through analysis of nine leading Virtual Fit platforms, the persona of a single female dress form was used to work through the customer journey. Through this, screen shot data captured along each stage in relation to the four research questions listed above. Following this, the study utilised content analysis structure with NVivo as a qualitative thematic analysis tool.

This study found that despite a large number of platforms using virtual fit technology, only a handful companies exist that provide such technology and interfaces; often based upon subjective 'previous purchases' rather than scientific prediction. This issue is made more complicated in how subjective measures such as personal perception of one's body is required (e.g. what size are you), besides body shape; a concept shown to be 'broken' and not fit-for-purpose. In addition, many of the technologies use limited and often misinterpreted body measurements, the impact of which is explored in greater detail within the paper. This study contributes to the understanding of the information required from users by virtual fit platforms, and the understanding of the output as presented by virtual fit platforms. The research goal is to contribute to knowledge as a potential guideline for any future projects in virtual fit and to help direct body scanning developments to better support these platforms.

Keywords: Virtual Fit, 3D Body Scanning, Computer Aided Tailoring, Interaction Design; eCommerce, Customisation, Fashion

\section{Introduction}

To unify the methodology of Virtual Fit platforms and allowing cross platform integration of 3D Body Scanning, the current Virtual Fit platforms need to be assessed in terms of their size recommendation approach and user interaction. The global fashion retail is worth over $\$ 212$ billion [3], but only $14.7 \%$ of the UK retail stems from e-commerce, $12 \%$ of which is from textiles, clothing and footwear stores, showing great uncapitalised potential in this sector [4]. The major issue with fashion Electronic Commerce (e-Commerce) dissatisfaction is related to not being able to assess the correct size and fit for the consumer's body [5]. Many retailers have turned to Virtual Fit platforms to give better guidance to consumers. However, this technology has yet to reach sufficient diffusion within society and achieve its full potential. To address this problem, this paper explores the current state of Virtual Fit platforms as a size and fit tool to assist users in the evaluation of garments in an e-Commerce context. 
The effectiveness of Virtual Fit platforms was first examined by McDonnell et al. [6] who conducted psychophysical experiments to tests of stiffness of virtual dress. In his result, participants showed better sorting performance with high detailed quality cloth than low detailed quality cloth. A similar test could be conducted to measure size discrimination performance using different qualities of simulation garments and in several sizes. Kim and LaBat [7] investigated the accuracy of virtual try-on technology for garment fit evaluation by having users compare the fit of virtual trousers to that of real trousers. Their findings addressed the aspects of virtual garments that need to be improved to fully represent real garment. Kim [8] also investigated the level of garment size variation that viewers can perceive with $3 \mathrm{D}$ virtual try-on technology, and how accurately the technology allows viewers to perceive the size difference with 3D virtual try-on technology. The results indicated that the 3D virtual try-on technology has the capacity of showing size variations clearly enough for viewers to perceive and discriminate size variations in the 3D virtual trousers as as small as $\pm 0.55 "$. Kim and Sundar [9] highlighted that by utilising realistic avatars in virtual environments allow users to see themselves from a third person perspective. Study of Shin and Baytar [10] researchers investigated effects of viewing models' bodies and body satisfaction on female consumers' concerns about garment fit and size and their intention to use virtual fit when shopping online. They found the degree to which the avatar resembles the actual vs. desired version of the user's self could be quite consequential for a number of attitudinal as well as behavioural outcomes. Harvey et al. [11] created a model for the processes aimed at gathering 3D models and measurements within in a customer's personalized database. He suggested that developing a software tool for the try-on involving digital garments and a virtual mannequin will provide tools for the apparel businesses to gain a competitive edge through custom-made garments.

The aim of this study is to understand the current state of Virtual Fit platforms to direct and develop fashion e-Commerce services enhanced by 3D Body Scanning. As a consequence, this study addresses three research objectives:

1. To understand the information required from users by Virtual Fit platforms because we need to assess the potential of 3D Body Scanning to connect with Virtual Fit technologies.

2. To understand the outputs as presented by Virtual Fit platforms because we need to facilitate the innovative application of anthropometric data in fashion e-Commerce when directing 3D Body Scanning developments for service provision.

3. To test how Virtual Fit platforms work out size and fit recommendations to allow more standardisation in assessments between individuals and against recognised criteria and find opportunities for 3D Body Scanning data to be integrated within fashion e-Commerce.

To address the research aims, this paper investigates the consumer journey in terms of data requirements, and information output from the leading Virtual Fit platforms through a qualitative analysis methodology. In answering the research objectives, the main contributions to the field of Service Design and consumer interaction made by this study are:

- Current Virtual Fit platforms are shown to produce non-compatible size and fit recommendations based on a multitude of anthropometric and self-reported body measurements, with little to no agreement on which are used within the systems; see Section 4.1. and 4.1.1.

- Existing 'bad habits' of consumers to buy incorrect fitting garments is sustained rather than addressed through Virtual Fit platforms, leading to the need for quantified garment fit guidelines integrated with 3D Body Scanning; see Section 4.1.2.

- There is a lack of lack of universal visual communication of size and fit between all Virtual Fit platforms, limiting the ability for collective acceptance of online size and fit recommendation platforms that requires a more user cantered and service design approach; see Section 4.2 . 


\section{Theoretical Background}

Advanced information technology and big data utilisation is on the rise and is very important for fashion companies to apply it effectively [12]. Digital content creation (e.g. online atmospherics) has become a major challenge in recent years as the virtual world of personal magic mirrors, Virtual Fit, and online customisation increases in size and importance. As stated in earlier research [13]-[15], the maturation of internet technology has bolstered e-Commerce's expansion beyond extensive product offerings, customer convenience, ease of navigation, and security that affect online marketing. Digital data, interactivity, and internet technology are changing the ways we interact in online shopping domain [16], [17]. In this domain, Virtual Fit technology has great potential to increase shopping engagement, enhance the product experience, and deliver better fitting garments [18].

Kartsounis et al. [19], highlighted how the impact of Virtual Fit platforms shall be significant for all segments of fashion retailing. This may lead to infrastructures and standards to support harmonisation and product description technologies to increase customer confidence in buying clothes online with the rise of virtual tailoring. In relation to this, several companies are already proposing solutions to size and fit issues. Current Virtual Fit platforms offer innovative tools for users to engage with apparel including virtual models, virtual catwalks, 360-degree views, apparel heat maps, and style recommendation libraries [20]. Academic approaches about size and fit offer a good framework both for standardising practice and for developing the techniques for systematic assessment of fit against recognisable criteria. Gill [18], created a classification method where Virtual Fit platforms are classified as 1) size recommendation, 2) fit recommendation, and 3) fit visualisation. $\mathrm{He}$ further elaborated, that all models need familiarity with methods of determining fit, from anthropometric classification through to the direct engagement with fit visualisation tools. These may appear to be abstract compared with traditional perceptions experienced when trying garments on, often driven by self-perception misconceptions [21], [22].

\section{Methodology}

To find the leading virtual fit platforms, virtual fit websites were discovered through snowball sampling, examining academic journals and fashion websites (WGSN, BOF-Business of Fashion, Just Style, Drapers) for the keywords 'virtual fit' and 'virtual try-on'. In order to be suitable for this study, websites had to be 1) embedded within a live fashion retail website, 2) be available via non-subscription platforms, and 3) be offered in an English language interface. In total, nine virtual fit websites were revealed, being associated with online fashion retailers in the high street and diffusion market segments; see Table 1.

Table 1 Virtual Fit Platforms

\begin{tabular}{l|ll}
\hline Virtual Fit Technology & Retailer name & URL address \\
\hline Fit Analytics & Tommy Hilfiger & {$[23]$} \\
Fit Predictor & Boden & {$[24]$} \\
Fits Me & Henri Lloyd & {$[25]$} \\
Metail & House of Holland & {$[26]$} \\
True Fit & Phase Eight & {$[27]$} \\
Virtusize & Filippa K & {$[28]$} \\
Belcurves & Mark and Spencer & {$[29]$} \\
StyleWhile & Seezona & {$[30]$} \\
Virtual Outfits & Yoox & {$[31]$} \\
\hline
\end{tabular}

The customer journey and the virtual fit options were explored by using each Virtual Fit platform to find the size and fit recommendations of a standardised body, which with this study was an Alvanon ${ }^{\mathrm{TM}}$ body form. To make sure the measurements of the body form were correct, the body form was scanned using a size stream 3D body scanner [32], a technology showed to be reliable for scientific research [33] for the measurements used within this study. Throughout the user journey, screen shots of the interface were captured using the MacOS [34] native screen printing, with images investigated through thematic content analysis with NVIVO [2] qualitative analysis software. The application of content analysis involves the classification of the information interfaces of virtual fit platforms into common meaning categories, measuring their importance in terms of their frequency of occurrence in the text, and then exploring the relationship between these relative frequencies [35]. 


\section{Results and Analysis}

\subsection{Information Requirements for Virtual Fit platforms}

To understand the range of information required by Virtual Fit platforms from users to receive the size and fit recommendation, Open Coding was applied to the user journey of captured data. Through this, two major categories of information were discovered:

a) Anthropometric Data - Describing body measurements which users were ask to provide for the garment size/fit calculation.

b) Garment Data - Describing the past garment purchase, fit preferences, and style preferences to provide size/fit recommendations.

\subsubsection{Anthropometric Data Requirements}

Table 2 presents the range of anthropometric data required of the user for each Virtual Fit platforms, along with the frequency of occurrence within thematic analysis. The key finding here is that despite all Virtual Fit platforms having similar objectives of fit and style recommendation, there was little to no agreement amongst the nine platforms on the 17 pieces of anthropometric data required to calculate size and fit.

Table 2 Anthropometric Data

\begin{tabular}{|c|c|c|c|c|c|c|c|c|c|}
\hline & A : Belcurves & B : Fit Analytics & C : Fit Predictor & $\mathrm{D}:$ Fits $\mathrm{Me}$ & E : Metail & F : Stylewhile & G : True Fit & H: Virtual Outfits & I: Virtusize \\
\hline $1:$ Age & 0 & 1 & 0 & 1 & 0 & 0 & 3 & 0 & 0 \\
\hline 2: Arm & 1 & 0 & 0 & 0 & 0 & 1 & 0 & 0 & 0 \\
\hline 3 : Belly Shape & 0 & 1 & 0 & 0 & 0 & 0 & 1 & 0 & 0 \\
\hline 4 : Body Shape & 0 & 0 & 0 & 1 & 0 & 0 & 0 & 2 & 0 \\
\hline 5 : Bra Size & 0 & 2 & 0 & 1 & 1 & 0 & 2 & 0 & 0 \\
\hline $6:$ Bust Size & 1 & 0 & 0 & 0 & 0 & 1 & 0 & 1 & 0 \\
\hline 7 : Calves & 0 & 0 & 0 & 0 & 0 & 0 & 1 & 0 & 0 \\
\hline $8:$ Face & 0 & 0 & 0 & 0 & 1 & 0 & 0 & 2 & 0 \\
\hline 9: Foot Arch & 0 & 0 & 0 & 0 & 0 & 0 & 1 & 0 & 0 \\
\hline 10 : Height & 1 & 1 & 0 & 1 & 1 & 0 & 3 & 1 & 0 \\
\hline 11 : Hip Shape & 1 & 1 & 0 & 0 & 1 & 1 & 2 & 1 & 0 \\
\hline 12 : Inside Leg & 0 & 0 & 0 & 0 & 0 & 1 & 2 & 0 & 0 \\
\hline 13 : Shoulders & 0 & 0 & 0 & 0 & 0 & 1 & 0 & 0 & 0 \\
\hline 14 : Skin colour & 0 & 0 & 0 & 0 & 0 & 0 & 0 & 1 & 0 \\
\hline 15 : Torso & 0 & 0 & 0 & 0 & 0 & 0 & 2 & 0 & 0 \\
\hline 16 : Waist & 1 & 0 & 0 & 0 & 1 & 1 & 0 & 1 & 0 \\
\hline 17 : Weight & 0 & 1 & 0 & 1 & 1 & 0 & 3 & 0 & 0 \\
\hline
\end{tabular}

The findings of Table 2 suggest a mismatch in sizing methodologies with no industry standardisation of how fit and style recommendations are based, which output result not comparable. The most prevalent anthropometric data utilised by the nine Virtual Fit platforms were height and hip shape. However, within each measurement, the inconsistency to pattern methodologies appeared as each platform referred to a different placement definition on a body.

Inconsistencies in the anthropometric data requirements are further confounded by when the users were ask for details about their body shape (e.g. belly, hip, shoulders) the platforms assumed the user had a enough understanding to classify their own body against loose criteria (e.g. narrow shoulders, curvy belly). As no universal descriptors for body shape and proportion exist, this subjective data has the potential to be inaccurate, meaning that fit and style recommendations based upon this have an added erroneous influence on the outputs.

\subsubsection{Garment Data Requirements}

Table 3 presents the range of garment data required of the user for each Virtual Fit platforms, along with the frequency of occurrence within thematic analysis. The key finding here is that information from users' personal wardrobe (representing past purchases and current fit preference) is used to work out size and fit. This provides Virtual Fit platforms with contextualised and meaningful data on users' shopping habits, as well as brand and size preferences. 
Table 3 Garment Data

\begin{tabular}{|c|c|c|c|c|c|c|c|c|c|}
\hline & A : Belcurves & B : Fit Analytics & C : Fit Predictor & D : Fits Me & E : Metail & $\mathrm{F}$ : Stylewhile & G : True Fit & $\mathrm{H}$ : Virtual Outfits & I: Virtusize \\
\hline 1: Past Purchase History & 0 & 0 & 2 & 0 & 0 & 0 & 16 & 0 & 3 \\
\hline 2 : Prefered Fit & 0 & 1 & 0 & 0 & 1 & 0 & 2 & 0 & 0 \\
\hline 3 : Prefered Style & 1 & 0 & 0 & 0 & 0 & 0 & 4 & 0 & 0 \\
\hline
\end{tabular}

Unlike the anthropometric data requirements that spanned 17 distinct measurements, only three attitudes on garment preferences were required by the nine platforms:

a) Past Purchase History - Users can create an account based on their email address and is expected to answer questions related to their earlier shopping purchases. This gives retailers ideas about which brands and sizes variations user shop and tailor specific product recommendations to user email inbox.

b) Preferred Fit - Perception of good fit for a consumer, this may range from a desire for a garment to conform loosely to the body (i.e. give comfort) to perfect conformation to the body (i.e. give maximum form appearance).

c) Preferred Style - Style ease and the amount of fullness added to garments to create a desired visual effect. Style ease often results in added volume to certain body areas, depending upon personal preference and current fashion in various clothing categories, e.g. fabric that stretches could be comfortable even if it measures less than body measurement; in the design of harem pants, more fabric and the oversize feel is acceptable.

Considering use of garment fit data, there was no strong bias in the Virtual Fit platforms to use one method over another: Past Purchase $(n=3)$; Preferred Fit $(n=3)$; Preferred Style $(n=2)$. However, Table 3 shows that True Fit has a high dependency upon Past Purchase History, being the only technology with a strong bias towards one method over another. True fit also uses multiple methods, unlike other technologies that use single methods.

\subsection{Information Outputs from Virtual Fit platforms}

To understand the visual style and fit outputs from Virtual Fit platforms, the ten key variables of the size/fit recommendation were identified through thematic analysis Table 4 . The key finding here is a lack of universal visual communication of size and fit between all nine of the Virtual Fit platforms.

Table 4 Virtual Fit Visualisation Outputs relative to Virtual Fit Platforms

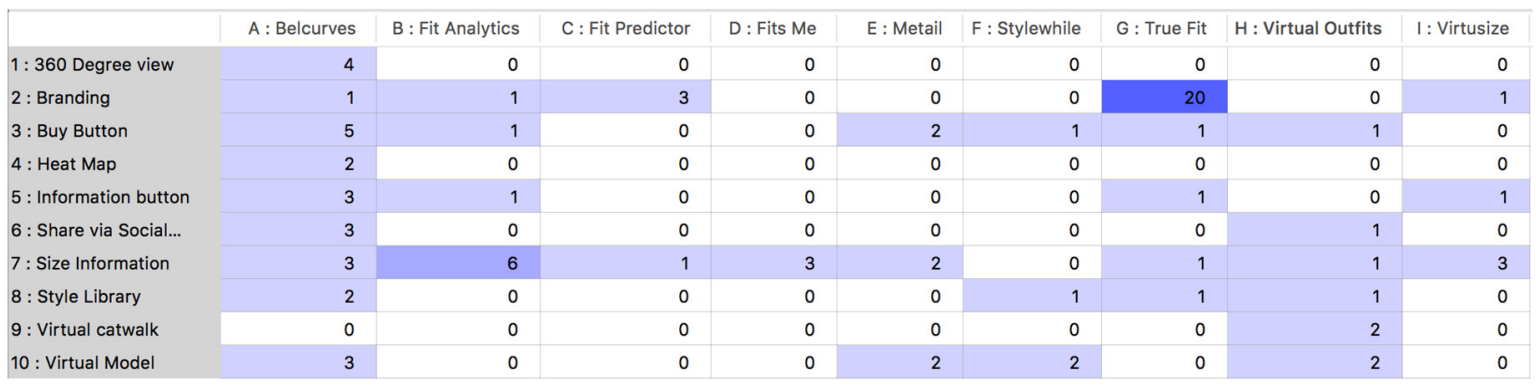

The most common variable across eight of the Virtual Fit platforms was size prediction. The sizes were presented to users in different sizing metrics, which were applied by each retailer. Therefore, output size information is not compatible. Even so, the only platform that does not give size or fit information is Stylewhile. Instead, this platform focuses on the visualisation aspect of garment style on a personalised virtual model. The element of personalisation is used to allow the creation and manipulation of interactivity and telepresence to simulate actual experience with the product.

While anthropometric visualisations were a key element of the visual output, the Virtual Fit platforms integrate key design elements from User Experience (UX) design to enhance the retailers brand image and provide a persuasive retail environment. The branding element $(n=7)$ was applied consistently to link marketing elements within platform. An online brand development strategy focuses on increasing emphasis on brand experience with application of interactive tools such as style library $(n=5)$, virtual model $(n=4)$, virtual catwalk $(n=1)$, heat map $(n=1)$, and 360-degree view $(n=1)$. However, no consistent methods to present proposed closeness of fit exist, and these visual methods requires consumers to develop the skills on how to align feedback in a meaningful way. 


\section{Discussion}

Anthropometric data required from users was shown in Section 4.1. to have a limited connection to measurements that garment construction needs. Because of this, there is no consistent connection between Virtual Fit predictions of garment size and pattern cutting or pattern grading practices that garments are based on. This concept is not unique to Virtual Fit platforms and is discussed further within these conference proceedings [36]. This finding agrees with Gill [18] as comparison between systems for Virtual Fit analysis shows the existence of different degrees of understanding of the potential variables in clothing fit. Moreover, the research by Ashdown and O'Connell [37] suggested customers do not possess full knowledgeable about what forms a good fit. To address this, Virtual Fit platforms can educate users with tag information describing special fit features of a style, or with information on good and bad fit.

Body Shape Self-perceptions are shown in Section 4.1.1. to be used by Virtual fit platforms to predict the relationships between dimensions or regions of the body. To achieve this, Virtual fit needs to be supported by a sound theoretical understanding of the relationships between shape, size, and fit [18]. As the public has limited understanding on classifying their own body shape [21], [22], Body Scanning has the potential to be a major facilitator in directing suitable garment fit. As suggested by Ashdown and O'Connell [37], one dimensional measurements also may not predict garment fit with sufficient accuracy. Given the wide range of body proportions, shapes, and postures, interactions among measurements may cause misfits that cannot be identified with single linear measurement comparisons.

Previous garment purchase information is shown in Section 4.1.2. to be considered a key driver of size recommendations in Virtual Fit platforms. However, earlier research [38], [39] suggests that few consumers understand the dynamics of sizing and fit responsible for creating the modern apparel sizing systems. Besides this, Ashdown and O'Connell found [37] that the user's subjective measure did not correspond with garment fit evaluation by experienced professionals. This is because during the manual measurement process the human body is often compressed. The amount of tension applied depends on the subjective assessment of the person conducting the measurement and therefore cannot be standardised [40]. As a consequence, some of the most utilised data in recommending 'correct' garment fit has a high degree of potential error. This leads to consumers purchasing 'incorrect' sizes again and again rather than achieving 'perfect' fit as promised by the technology. This is an opportunity for 3D Body Scanning to enhance fit recommendations due to the reliable non-ambiguity of the technology [33], if style and fit can be quantified [41].

Section 4.2. evaluated the visual output which users receive after sharing their anthropometric and garment purchase information. However the major result was the lack of universal visual communication of size and fit between all Virtual Fit platforms. Development of visual tools needs to have a clear purpose to the user, and since size and fit are often comparative experiences, comparisons must be made in a meaningful way. This finding is consistent with Gill [18] as the ability to realise the 3D/2D nature of the body and pattern relationships must support integration through technology. This development requires new methods with the ability to recognise fit in terms of body and garment relationships and interactions. 3D Body Scanning allows for more linearity between the individual, the product development process, and the eventual garment and fit assessment.

\section{Conclusion}

This paper set out to explore how Virtual Fit platforms approach user interaction in terms of information requirements, recommendation outputs, and size and fit calculations. This paves the way for Virtual Fit platform unification, and show where 3D Body Scanning may integrate with such ergonomic prediction tools.

The main contribution of this paper is that current Virtual Fit platforms are unlikely to be compatible or relate to the theory that determines actual garment size. Because of this, 3D Body Scanning has a great potential to enrich the accuracy of garment size and fit prediction over and above current Virtual Fit offerings.

The implications of this paper relate primarily to Virtual Fit developers, pattern cutters, and 3D Body Scanning manufacturers. These key groups who must work together to achieve the visual of accurate, appropriate, and suitable size and fit prediction. For platform developers, this paper directs strategies to include 3D Body Scanning. This will need necessary anthropometric measurement identification 
from scans and the development of size and fit protocols that increase reliability and validity of size and fit predictions. For pattern cutters, as current Virtual Fit platforms do not clearly link to body shape and proportions, new methods for creating patterns from anthropometric analysis must integrate into existing practices. This is to help manage the change to more automated and precise systems of mass customisation and virtual tailoring. As the advantages of 3D Body Scanning for fit analysis received more recognition, the technology will contribute to better fitting garments. 3D Body Scanning should also lead to and higher e-Commerce profitability through lower return rates and more efficient supply chain management.

The limitation of this paper is the small sample size of Virtual Fit platforms. However, this is due to the newness of the technology only having a handful of offerings when non-English language and nonweb based Virtual Fit platforms are excluded.

Future research will open up Virtual Fit assessments to the wider range of international and non-web based platforms, including Japanese and French garment fit prediction services. Additional research will focus on the consumer acceptance of 3D Body Scanning to lead to greater acceptance of the technology in the high street retailers. Also, research must focus on 3D Body Scanning as a service to be designed for non-expert fashion consumers with associated profitable business models; possibly through co-design methodologies [42].

\section{References}

[1] J. Kim and S. Forsythe, "Adoption of virtual try-on technology for online apparel shopping," J. Interact. Mark., vol. 22, no. 2, pp. 45-59, 2008.

[2] QSR, "NVivo 11." QSR International, Melbourne, Australia, 2016.

[3] K. McCarthy, B. Perkins, N. Pope, L. Portaluppi, V. Scaramuzzi, and L. Su, "Global Powers of Luxury Goods 2017," Westlake, USA, 2017.

[4] Mintel, "Online Retailing - UK - July 2017," Mintel, 2017. [Online]. Available: http://academic.mintel.com/display/793487/. [Accessed: 22-Aug-2017].

[5] Mintel, "Online Retailing," 2016.

[6] R. Mcdonnell, S. Dobbyn, S. Collins, and C. O'Sullivan, "Perceptual evaluation of LOD clothing for virtual humans," Proc. ACM SIGGRAPH/Eurographics Symp. Comput. Animat., pp. 117126, 2006.

[7] D.-E. Kim and K. LaBat, "An exploratory study of users' evaluations of the accuracy and fidelity of a three-dimensional garment simulation," Text. Res. J., vol. 83, no. 2, pp. 171-184, 2013.

[8] D. E. Kim, "Psychophysical testing of garment size variation using three-dimensional virtual try-on technology," Text. Res. J., vol. 86, no. 4, pp. 365-379, 2016.

[9] Y. Kim and S. S. Sundar, "Visualizing ideal self vs. actual self through avatars: Impact on preventive health outcomes," Comput. Human Behav., vol. 28, no. 4, pp. 1356-1364, 2012.

[10] E. Shin and F. Baytar, "Apparel Fit and Size Concerns and Intentions to Use Virtual Try-On: Impacts of Body Satisfaction and Images of Models' Bodies," Cloth. Text. Res. J., vol. 32, no. 1, pp. 20-33, Jan. 2014.

[11] E. R. Harvey, J. Bellemare, D. Boulanger, I. Lessard, and P. Proulx, "3D Digital Technologies for Virtual Fitting of Garments in Tailor-Made Application," in 5th International Conference on 3D Body Scanning Technologies, 2014, no. October, pp. 399-405.

[12] T. L. Lewis and S. Loker, "Trying on the Future: Exploring Apparel Retail Employees Perspectives on Advanced In-Store Technologies," Fash. Pract., vol. 9370, no. January, pp. $1-25,2017$.

[13] A. M. Fiore and H. Jin, "Influence of image interactivity on approach responses towards an online retailer," Internet Res., vol. 13, no. 1, pp. 38-48, Mar. 2003.

[14] C. Liu, K. P. Arnett, and C. Litecky, "Design quality of websites for electronic commerce: Fortune 1000 webmasters' evaluations," Electron. Mark., vol. 10, no. 2, pp. 120-129, 2000.

[15] G. L. Lohse, S. Bellman, and E. J. Johnson, "Consumer buying behavior on the Internet: Findings from panel data," J. Interact. Mark., vol. 14, no. 1, pp. 15-29, Jan. 2000.

[16] S. Loker, S. Ashdown, and E. Carnrite, "Dress in the Third Dimension: Online Interactivity and Its New Horizons," Cloth. Text. Res. J., vol. 26, no. 2, pp. 164-176, Apr. 2008.

[17] C. J. Parker and H. Wang, "Examining hedonic and utilitarian motivations for m-commerce fashion retail app engagement," J. Fash. Mark. Manag. An Int. J., vol. 20, no. 4, pp. 487-506, Sep. 2016.

[18] S. Gill, "A review of research and innovation in garment sizing, prototyping and fitting," Text. Prog., vol. 47, no. 1, pp. 1-85, Jan. 2015. 
[19] G. a Kartsounis, N. M. Thalmann, and H. Rodrian, "E-TAILOR: Integration of 3D Scanners, CAD and Virtual-Try- on Technologies for Online Retailing of Made-to-Measure Garments," 1999.

[20] D.-E. Kim and K. Labat, "Consumer experience in using 3D virtual garment simulation technology," J. Text. Inst., vol. 104, no. 8, pp. 819-829, Aug. 2013.

[21] N. Som and S. Mukhopadhyay, "Body weight and body shape concerns and related behaviours among Indian urban adolescent girls," Public Health Nutr., vol. 18, no. 6, pp. 10751083, Apr. 2015.

[22] S. Kim and W.-Y. So, "Prevalence and sociodemographic trends of weight misperception in Korean adolescents," BMC Public Health, vol. 14, no. 1, p. 452, Dec. 2014.

[23] Tommy Hilfiger, "Tommy Hilfiger Home Page," Tommy Hilfiger, 2017. [Online]. Available: https://uk.tommy.com. [Accessed: 23-Aug-2017].

[24] Boden, "Boden Home Page," Retail Website, 2017. [Online]. Available: http://www.boden.co.uk. [Accessed: 20-Jul-2017].

[25] Henri Lloyd, "Henri Lloyd Home Page," Retail Website, 2017. [Online]. Available: https://www.henrilloyd.com. [Accessed: 20-Jul-2017].

[26] House of Holland, "House of Holland Home Page," Retail Website, 2017. [Online]. Available: https://www.houseofholland.co.uk. [Accessed: 20-Jul-2017].

[27] Phase Eight, "Phase Eight Home Page," Retail Website, 2017. [Online]. Available: http://www.phase-eight.com. [Accessed: 20-Jul-2017].

[28] Filippa K, "Filippa K Home Page," Retail Website, 2017. [Online]. Available: https://www.filippa-k.com. [Accessed: 20-Jul-2017].

[29] Belcurves Home Page, "Belcurves," Belcurves, 2017. [Online]. Available: http://www.belcurves.com/index.php?lang=en. [Accessed: 20-Jul-2017].

[30] Seezona, "Seezona Home Page," Retail Website, 2017. [Online]. Available: https://www.seezona.com. [Accessed: 20-Jul-2017].

[31] Virtual Outfits, "Virtual Outfits Home Page," Virtual Fit Website, 2017. [Online]. Available: http://www.virtualoutfits.com. [Accessed: 20-Jul-2017].

[32] SizeStream, "SS14 3D Body Scanner." SizeStream, Cary, NC, USA, 2017.

[33] C. J. Parker, S. Gill, and S. Hayes, "3D Body Scanning has Suitable Reliability: An Anthropometric Investigation for Garment Construction," in 8th International Conference and Exhibition on 3D Body Scanning and Processing Technologies, 2017.

[34] Apple, "MacOS Sierra." Apple, Cupertino, USA, 2016.

[35] F. Henri, "Computer conferencing and content analysis," in Collaborative Learning Through Computer Conferencing- The Najaden Papers., A. R. Kaye, Ed. Springer-Verlag Berlin Heidelberg, 1992, pp. 117-137.

[36] S. Gill, M. Ahmed, C. J. Parker, and S. G. Hayes, "Not All Body Scanning Measurements Are Valid: Perspectives from Pattern Practice," in 8th International Conference and Exhibition on 3D Body Scanning and Processing Technologies, 2017.

[37] S. P. Ashdown and E. K. O'Connell, "Comparison of Test Protocols for Judging the Fit of Mature Women's Apparel," Cloth. Text. Res. J., vol. 24, no. 2, pp. 137-146, Mar. 2006.

[38] S. Grogan, S. Gill, K. Brownbridge, S. Kilgariff, and A. Whalley, "Dress fit and body image: A thematic analysis of women's accounts during and after trying on dresses," Body Image, vol. 10, no. 3, pp. 380-388, 2013.

[39] S. Gill and K. Brownbridge, "The Truth of standard sizing," in In: 3rd Global Conference Beauty: Exploring Critical Issues., 2013.

[40] Hohenstien Institute and The Nottingham Trent University, "Task 4 . 1 European Sizing Survey Manual for Conducting standardised 3D Body Scan Surveys as Basis for International Anthropometric Database," no. December, 2002.

[41] E. Kirchdoerfer and J. Bougourd, "Comparison of Body Measurements eT - CLUSTER PROPOSED HUMAN BODY MEASUREMENTS STANDARD Confidential document IST2000-26084," no. D, pp. 1-66, 2002.

[42] V. Mitchell, T. Ross, A. May, R. Sims, and C. Parker, "Empirical investigation of the impact of using co-design methods when generating proposals for sustainable travel solutions," CoDesign, vol. 12, no. 4, pp. 205-220, Oct. 2016. 